\title{
Demographic, Behavioral, and Clinical Characteristics of the Ambulatory Diabetic Population of the Dominican Republic, 2018
}

\section{Leslie Anne Duncan, $\mathrm{MPH}^{1^{*}}$, Paige Hornsby, $\mathrm{PhD}^{1}$, Aaron Pannone, PhD ${ }^{1}$, Omar W Ebrahim ${ }^{2}$ and Ammar Ibrahim, $M D^{3}$}

${ }^{1}$ Department of Public Health Sciences, School of Medicine, University of Virginia, USA

${ }^{2}$ Universidad IberoAmericana, Santo Domingo, Dominican Republic

${ }^{3}$ Instituto Nacional de Diabetes, Endocrinología y Nutrición, Santo Domingo, Dominican Republic

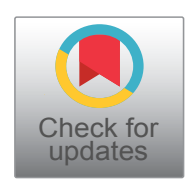

*Corresponding author: Leslie Anne Duncan, Department of Public Health Sciences, School of Medicine, University of Virginia, P.O. Box 800717, Charlottesville, VA 22098, USA

\begin{abstract}
Objectives: Type 2 diabetes has steadily increased in prevalence in the Dominican Republic. Our objectives were to identify the demographic, behavioral, and clinical characteristics of adult male and female ambulatory diabetic patients in the Dominican Republic to better understand diabetes in the Dominican population.

Methods: We conducted a retrospective chart review of a random sample of ambulatory patients treated at the diabetes clinic at the Instituto Nacional de Diabetes, Endocrinología y Nutrición (INDEN) whose first appointment was in 2018. We abstracted data for demographic, behavioral, and clinical variables and compared them by sex.

Results: Of the 500 patients, a slight majority were female. Over $84 \%$ of patients had Type 2 diabetes. Female patients had significantly higher rates of several comorbidities, including obesity and hypertension. Male patients were slightly younger than female patients, and had significantly higher rates of several symptoms. In addition, men had significantly higher levels of $\mathrm{HbA} 1 \mathrm{c}$. Over $50 \%$ of all patients had a high fasting blood glucose level. The majority of the population reported never exercising.

Conclusion: Sex-specific interventions are needed help to minimize the burden of the disease and improve the quality of life for the Dominican population. Programs to address high prevalence risk factors, like obesity and hypertension, and increase early access to medical care could help with prevention and/or management of diabetes, improve the health of the population, and decrease the economic burden of disease on both the population and the healthcare system of the Dominican Republic.
\end{abstract}

\section{Keywords}

Demographics, Diabetes mellitus type 2, Dominican republic, Risk factors, Retrospective studies

\begin{abstract}
Abbreviations
DR: Dominican Republic; LAC: Latin America and the Carribean; INDEN: National Institute of Diabetes, Endocrinology, and Nutrition; UNIBE: Universidad Iberoamericana; BMI: Body Mass Index; UTI: Urinary Tract Infection; HDL: High-Density Lipoprotein; LDL: Low-Density Lipoprotein
\end{abstract}

\section{Introduction}

In 2018, the International Diabetes Federation documented the prevalence of diabetes among adults aged 20-79 in the Dominican Republic (DR) to be $8.7 \%$ [1], one of the highest in Latin America and the Caribbean (LAC) [2]; however, unpublished data from the same year showed the prevalence as $13.5 \%$ [3]. In addition, age-standardized diabetes prevalence in the adult population in the DR has steadily increased since 1980 [4]. In 2016, diabetes was responsible for $4 \%$ of all deaths in the DR [4]. The actual magnitude of the problem is likely much higher, as it is estimated that $24-50 \%$ of adults with diabetes are undiagnosed in LAC [2]. Much of the increased rate of Type 2 diabetes is due to the epidemiological transition from infectious diseases to chronic conditions, which has changed

Citation: Duncan LA, Hornsby P, Pannone A, Ebrahim OW, Ibrahim A (2021) Demographic, Behavioral, and Clinical Characteristics of the Ambulatory Diabetic Population of the Dominican Republic, 2018. Int J Diabetes Clin Res 8:143. doi.org/10.23937/2377-3634/1410143

Accepted: May 29, 2021: Published: May 31, 2021

Copyright: (C) 2021 Duncan LA, et al. This is an open-access article distributed under the terms of the Creative Commons Attribution License, which permits unrestricted use, distribution, and reproduction in any medium, provided the original author and source are credited. 
the health profile of the DR [5]. The urbanization and industrialization of the country have contributed to lifestyle changes, resulting in poor quality diets as well as reduced physical activity [2].

Healthcare costs among those with diabetes (all types) are two to three times higher than those without diabetes [6]. In 2000, in the DR, the cost of diabetes was estimated to be 625.1 million US\$ [6]. The total indirect cost of diabetes was estimated to be 399.4 million US\$, well above the total direct cost of 225.7 million US\$, suggesting that complications, comorbidities, and disability contribute substantially to costs for the diabetic population [6]. In addition, the per capita direct cost of diabetes in the DR was 888 US\$, one of the highest in LAC [6]. In 2018, the average diabetes-related cost per person with diabetes was 1,502.2 US\$ [1].

Despite the importance of this emerging public health issue, little research has focused on diabetes in the DR $[7,8]$. We identified demographic, clinical, and behavioral characteristics of male and female ambulatory diabetic patients treated in the diabetes clinic at the national diabetes hospital to better understand diabetes in the Dominican population.

\section{Subjects, Materials, Methods}

We conducted a retrospective chart review of ambulatory patients of the diabetes clinic at the Instituto Nacional de Diabetes, Endocrinologia, y Nutricion (INDEN) in Santo Domingo, DR in the summer of 2019.

Patients over the age of 18 whose first diabetes clinic appointment was between January 1, 2018 and December 31, 2018 were eligible for the study. We chose 2018 because it was the first year that ambulatory patient information was recorded in an electronic medical record and it was the most recently completed year. Electronic medical records were available for 5,716 patients. To select a random sample from among these patients, we assigned each patient a random number from 0 to 1 in Excel. We then sorted the patients from smallest to largest based on the random number and checked each patient for the inclusion criteria. Patients were excluded if their electronic chart contained no data or if their first appointment was not in 2018. One patient was excluded once it became clear that her identity had been stolen and the clinical information was from two different people. A total of 34 patients were excluded.

We developed a data abstraction form after reviewing charts and with the input of resident physicians. We pilot tested the form on twenty charts, taking note of how the information on symptoms and comorbidities was recorded, and adjusted the form for accuracy.

We abstracted data on age, sex, residence, occupation, exercise status, smoking status, alcohol use, type and length of diabetes, current treatment, frequency of clinic visits, family history of diabetes, diabetes-related hospitalizations, comorbidities, height, weight, blood glucose, $\mathrm{HbA1c}$, lab tests, and symptoms. For blood glucose and $\mathrm{HbA1c}$, we abstracted all values recorded in the chart along with the date. For the lab tests, only the most recent value was recorded along with the date. For current treatment, the most recent treatment was recorded along with whether there was a change in medication from the initial visit.

For continuous variables, we calculated the median and interquartile range. For categorical variables, we calculated the frequency and percentage for each category. All data are presented for the total population as well as stratified by sex. For variables with multiple recorded responses, like weight or HbA1c, the most recent measurement was used. For all statistical analyses, SAS version 9.4 was employed. $P$-values were calculated for categorical variables and continuous variables using chi-square tests and t-tests, respectively; all $P$-values were two-tailed, and $P<0.05$ was considered statistically significant.

Our research protocol was approved by the Universidad Iberoamericana's (UNIBE) ethics committee and the University of Virginia's Human Subject Research Institutional Review Board. No identifying data were collected for any patient.

\section{Results}

Demographic and behavioral characteristics of the patient population are presented in Table 1. A slight majority of the patients were female (55.80\%) and most lived in an urban area (91.89\%). The majority of the male patients were employed (73.60\%) while a slight majority of the female patients were homemakers (54.43\%). Nearly two-thirds of the patient population (65.87\%) reported no exercise at all. The male patients reported significantly more alcohol consumption than their female counterparts.

The clinical characteristics of the patient population are presented in Table 2 . Over $84 \%$ of the patients had Type 2 diabetes. The majority of the patients had a first degree relative with diabetes $(71.73 \%)$, and $11.80 \%$ of the population had been hospitalized due to a diabetesrelated reason. However, among patients with a debut of diagnosis, the rate of hospitalization was $8.3 \%$ compared to $14.6 \%$ for the others (data not shown). In addition, at least $28.4 \%$ of the population used some form of insulin treatment. Just over $75 \%$ of the patients visited the clinic twice or more per year.

Table 3 presents the comorbidities of the patient population. Over three-quarters of patients were overweight or obese, with over one-third (39.36\%) being obese. There was a statistically significant difference in body mass index (BMI) by sex, with more women in the obese category than men. More than half the patients reported having hypertension (56.40\%), with females having a higher rate than males $(64.16 \%$ 
Table 1: Demographic characteristics of INDEN ambulatory patients, 2018.

\begin{tabular}{|c|c|c|c|c|}
\hline & $\begin{array}{l}\text { Total Population } \\
n=500\end{array}$ & $\begin{array}{l}\text { Male } \\
n=221(44.2 \%)\end{array}$ & $\begin{array}{l}\text { Female } \\
n=279(55.8 \%)\end{array}$ & $P$-value \\
\hline Age (years) & $54(45,64)$ & $53(44,64)$ & $55(45,64)$ & 0.7479 \\
\hline $\begin{array}{l}\text { Residence } \\
\text { Urban } \\
\text { Rural } \\
\text { Other Country }\end{array}$ & $\begin{array}{l}(n=444) \\
408(91.89 \%) \\
33(7.43 \%) \\
3(0.68 \%)\end{array}$ & $\begin{array}{l}(n=205) \\
191(93.17 \%) \\
13(6.34 \%) \\
1(0.49 \%)\end{array}$ & $\begin{array}{l}(n=239) \\
217(90.79 \%) \\
20(8.37 \%) \\
2(0.84 \%)\end{array}$ & 0.6451 \\
\hline $\begin{array}{l}\text { Occupation } \\
\text { Employed } \\
\text { Unemployed } \\
\text { Retired } \\
\text { Homemaker } \\
\text { Student }\end{array}$ & $\begin{array}{l}(n=434) \\
227(52.30 \%) \\
43(9.91 \%) \\
25(5.76 \%) \\
130(29.95 \%) \\
9(2.07 \%)\end{array}$ & $\begin{array}{l}(n=197) \\
145(73.60 \%) \\
30(15.23 \%) \\
18(9.14 \%) \\
1(0.51 \%) \\
3(1.52 \%)\end{array}$ & $\begin{array}{l}(n=237) \\
82(34.60 \%) \\
13(5.49 \%) \\
7(2.95 \%) \\
129(54.43 \%) \\
6(2.53 \%)\end{array}$ & $<0.0001$ \\
\hline $\begin{array}{c}\text { Exercise } \\
\text { None } \\
\text { Any }\end{array}$ & $\begin{array}{l}(n=416) \\
274(65.87 \%) \\
142(34.13 \%)\end{array}$ & $\begin{array}{l}(n=176) \\
113(64.20 \%) \\
63(35.80 \%)\end{array}$ & $\begin{array}{l}(n=240) \\
161(67.08 \%) \\
79(32.92 \%)\end{array}$ & 0.5407 \\
\hline $\begin{array}{l}\text { Smoking } \\
\text { Never } \\
\text { Former } \\
\text { Current } \\
\text { Chewing Tobacco }\end{array}$ & $\begin{array}{l}(n=259) \\
158(61.00 \%) \\
75(28.96 \%) \\
25(9.65 \%) \\
1(0.39 \%)\end{array}$ & $\begin{array}{l}(n=131) \\
76(58.02 \%) \\
44(33.59 \%) \\
10(7.63 \%) \\
1(0.76 \%)\end{array}$ & $\begin{array}{l}(n=128) \\
82(64.06 \%) \\
31(24.22 \%) \\
15(11.72 \%) \\
0(0.0 \%)\end{array}$ & 0.2171 \\
\hline $\begin{array}{l}\text { Alcohol } \\
\text { Never } \\
\text { Former } \\
\text { Rarely } \\
\text { Sometimes } \\
\text { Always }\end{array}$ & $\begin{array}{l}(n=408) \\
204(50.00 \%) \\
35(8.58 \%) \\
3(0.74 \%) \\
152(37.25 \%) \\
14(3.43 \%)\end{array}$ & $\begin{array}{l}(n=196) \\
67(34.18 \%) \\
24(12.24 \%) \\
1(0.51 \%) \\
92(46.94 \%) \\
12(6.12 \%)\end{array}$ & $\begin{array}{l}(n=212) \\
137(64.62 \%) \\
11(5.19 \%) \\
2(0.94 \%) \\
60(28.30 \%) \\
2(0.94 \%)\end{array}$ & $<0.0001$ \\
\hline
\end{tabular}

Table 2: Clinical characteristics of INDEN ambulatory patients, 2018.

\begin{tabular}{|c|c|c|c|c|}
\hline & $\begin{array}{l}\text { Total Population } \\
n=500\end{array}$ & $\begin{array}{l}\text { Male } \\
n=221(44.2 \%)\end{array}$ & $\begin{array}{l}\text { Female } \\
n=279(55.8 \%)\end{array}$ & $P$-value \\
\hline $\begin{array}{l}\text { Type of Diabetes } \\
\text { Type } 1 \\
\text { Type } 2 \\
\text { Hyperinsulinemia } \\
\text { Gestational } \\
\text { Pre-Diabetes } \\
\text { Missing Diagnosis } \\
\text { Other }\end{array}$ & $\begin{array}{l}3(0.60 \%) \\
421(84.20 \%) \\
1(0.20 \%) \\
2(0.40 \%) \\
23(4.60 \%) \\
46(9.20 \%) \\
4(0.80 \%)\end{array}$ & $\begin{array}{l}1(0.45 \%) \\
183(82.81 \%) \\
1(0.45 \%) \\
0(0.0 \%) \\
10(4.52 \%) \\
25(11.31 \%) \\
1(0.45 \%)\end{array}$ & $\begin{array}{l}2(0.72 \%) \\
238(85.30 \%) \\
0(0.0 \%) \\
2(0.72 \%) \\
13(4.66 \%) \\
21(7.53 \%) \\
3(1.08 \%)\end{array}$ & 0.4706 \\
\hline $\begin{array}{l}\text { Diagnosis Length } \\
\text { Debut } \\
\leq 1 \text { year } \\
\text { 1-5 years } \\
\text { 5-10 years } \\
\text { 10-15 years } \\
\text { 15-20 years } \\
\text { 20-30 years } \\
\text { 30+ years } \\
\text { Not Applicable }\end{array}$ & $\begin{array}{l}(n=498) \\
145(29.12 \%) \\
82(16.47 \%) \\
89(17.87 \%) \\
63(12.66 \%) \\
41(8.23 \%) \\
22(4.42 \%) \\
9(1.81 \%) \\
2(0.40 \%) \\
45(9.04 \%)\end{array}$ & $\begin{array}{l}(n=220) \\
60(27.27 \%) \\
38(17.27 \%) \\
35(15.91 \%) \\
26(11.82 \%) \\
17(7.73 \%) \\
15(6.82 \%) \\
4(1.82 \%) \\
1(0.45 \%) \\
24(10.91 \%)\end{array}$ & $\begin{array}{l}(n=278) \\
85(30.58 \%) \\
44(15.83 \%) \\
54(19.42 \%) \\
37(13.31 \%) \\
24(8.63 \%) \\
7(2.52 \%) \\
5(1.80 \%) \\
1(0.36 \%) \\
21(7.55 \%)\end{array}$ & 0.3860 \\
\hline
\end{tabular}




\begin{tabular}{|c|c|c|c|c|}
\hline $\begin{array}{l}\text { Current Treatment } \\
\text { Basal Insulin } \\
\text { Basal + Prandial } \\
\text { Oral Antidiabetics } \\
\text { Combination } \\
\text { None }\end{array}$ & $\begin{array}{l}122(22.40 \%) \\
30(6.00 \%) \\
171(34.20 \%) \\
129(25.80 \%) \\
58(11.60 \%)\end{array}$ & $\begin{array}{l}60(27.15 \%) \\
14(6.33 \%) \\
72(32.58 \%) \\
54(24.43 \%) \\
21(9.50 \%)\end{array}$ & $\begin{array}{l}52(18.64 \%) \\
16(5.73 \%) \\
99(35.48 \%) \\
75(26.88 \%) \\
37(13.26 \%)\end{array}$ & 0.1879 \\
\hline $\begin{array}{l}\text { Visits per person } \\
\text { Once or more/mo. } \\
\text { More than 4x a yr. } \\
\text { 3-4x a year } \\
\text { Twice a year } \\
\text { Once a year } \\
\text { Only initial visit }\end{array}$ & $\begin{array}{l}3(0.60 \%) \\
100(20.00 \%) \\
134(26.80 \%) \\
142(28.40 \%) \\
0(0.0 \%) \\
121(24.20 \%)\end{array}$ & $\begin{array}{l}0(0.0 \%) \\
41(18.55 \%) \\
66(29.86 \%) \\
55(24.89 \%) \\
0(0.0 \%) \\
59(26.70 \%)\end{array}$ & $\begin{array}{l}3(1.08 \%) \\
59(21.15 \%) \\
68(24.37 \%) \\
87(31.18 \%) \\
0(0.0 \%) \\
62(22.22 \%)\end{array}$ & 0.1401 \\
\hline $\begin{array}{l}\text { First Degree Relative } \\
\text { Yes } \\
\text { No }\end{array}$ & $\begin{array}{l}(n=428) \\
307(71.73 \%) \\
121(28.27 \%)\end{array}$ & $\begin{array}{l}(n=195) \\
136(69.74 \%) \\
59(30.26 \%)\end{array}$ & $\begin{array}{l}(n=233) \\
171(73.39 \%) \\
62(26.61 \%)\end{array}$ & 0.4040 \\
\hline $\begin{array}{l}\text { Second Degree Relative } \\
\text { Yes } \\
\text { No }\end{array}$ & $\begin{array}{l}(n=428) \\
173(40.42 \%) \\
255(59.58 \%)\end{array}$ & $\begin{array}{l}(n=195) \\
74(37.95 \%) \\
121(62.05 \%)\end{array}$ & $\begin{array}{l}(n=233) \\
99(42.49 \%) \\
134(57.51 \%)\end{array}$ & 0.3404 \\
\hline $\begin{array}{l}\text { Hospitalized - Yes } \\
\text { Reason } \\
\text { Amputation } \\
\text { Diabetes Control } \\
\text { Diabetes Debut } \\
\text { Edema } \\
\text { Foot Ulcer } \\
\text { Heart Attack/Pre } \\
\text { Heart Surgery } \\
\text { High Blood Pressure } \\
\text { Ketoacidosis } \\
\text { Lesion/abscess } \\
\text { Pulmonary Edema/Embolism } \\
\text { Stroke }\end{array}$ & $\begin{array}{l}59(11.80 \%) \\
3(5.08 \%) \\
28(47.46 \%) \\
4(6.78 \%) \\
2(3.39 \%) \\
2(3.39 \%) \\
5(8.47 \%) \\
1(1.69 \%) \\
8(13.56 \%) \\
2(3.39 \%) \\
2(3.39 \%) \\
2(3.39 \%) \\
9(15.25 \%)\end{array}$ & $\begin{array}{l}28(12.67 \%) \\
2(7.14 \%) \\
12(42.86 \%) \\
2(7.14 \%) \\
2(7.14 \%) \\
2(7.14 \%) \\
2(7.14 \%) \\
1(3.57 \%) \\
4(14.29 \%) \\
1(3.57 \%) \\
1(3.57 \%) \\
2(7.14 \%) \\
4(14.29 \%)\end{array}$ & $\begin{array}{l}31(11.11 \%) \\
1(3.23 \%) \\
16(51.61 \%) \\
2(6.45 \%) \\
0(0.0 \%) \\
0(0.0 \%) \\
3(9.68 \%) \\
0(0.0 \%) \\
4(12.90 \%) \\
1(3.23 \%) \\
1(3.23 \%) \\
0(0.0 \%) \\
5(16.13 \%)\end{array}$ & 0.5916 \\
\hline
\end{tabular}

Table 3: Comorbidities of INDEN ambulatory patients, 2018.

\begin{tabular}{|c|c|c|c|c|}
\hline & $\begin{array}{l}\text { Total Population } \\
n=500\end{array}$ & $\begin{array}{l}\text { Male } \\
n=221(44.2 \%)\end{array}$ & $\begin{array}{l}\text { Female } \\
n=279(55.8 \%)\end{array}$ & $P$-value \\
\hline $\begin{array}{l}\text { BMI } \\
\text { Underweight } \\
\text { Normal } \\
\text { Overweight } \\
\text { Obese }\end{array}$ & $\begin{array}{l}(n=437) \\
14(3.20 \%) \\
93(21.28 \%) \\
158(36.16 \%) \\
172(39.36 \%)\end{array}$ & $\begin{array}{l}(n=191) \\
10(5.24 \%) \\
42(21.99 \%) \\
82(42.93 \%) \\
57(29.84 \%)\end{array}$ & $\begin{array}{l}(n=246) \\
4(1.63 \%) \\
51(20.73 \%) \\
76(30.89 \%) \\
115(46.75 \%)\end{array}$ & 0.0009 \\
\hline Hypertension & $282(56.40 \%)$ & $103(46.61 \%)$ & $179(64.16 \%)$ & $<0.0001$ \\
\hline Cardiovascular Disease & $32(6.40 \%)$ & $16(7.24 \%)$ & $16(5.73 \%)$ & 0.4947 \\
\hline Stroke & $25(5.00 \%)$ & $5(2.26 \%)$ & $20(7.17 \%)$ & 0.0124 \\
\hline Peripheral Vascular Disease & $33(6.60 \%)$ & $10(4.52 \%)$ & $23(8.24 \%)$ & 0.0962 \\
\hline Dyslipidemia & $84(16.80 \%)$ & $39(17.65 \%)$ & $45(16.13 \%)$ & 0.6521 \\
\hline Hypercholesterolemia & $79(15.80 \%)$ & $26(11.76 \%)$ & $53(19.00 \%)$ & 0.0277 \\
\hline $\begin{array}{l}\text { Non-Alcoholic Fatty } \\
\text { Liver Disease }\end{array}$ & $114(22.80 \%)$ & $41(18.55 \%)$ & $73(26.16 \%)$ & 0.0439 \\
\hline Thyroid disease/nodules & $62(12.40 \%)$ & $8(3.62 \%)$ & $54(19.35 \%)$ & $<0.0001$ \\
\hline UTI & $72(14.40 \%)$ & $18(8.14 \%)$ & $54(19.35 \%)$ & 0.0004 \\
\hline Edema & $78(15.60 \%)$ & $29(13.12 \%)$ & $49(17.56 \%)$ & 0.1741 \\
\hline
\end{tabular}

BMI: Body Mass Index; UTI: Urinary Tract Infection. 
Table 4: Lab results of INDEN ambulatory patients, 2018.

\begin{tabular}{|c|c|c|c|c|}
\hline & $\begin{array}{l}\text { Total Population } \\
\mathrm{n}=\mathbf{5 0 0}\end{array}$ & $\begin{array}{l}\text { Male } \\
n=221(44.2 \%)\end{array}$ & $\begin{array}{l}\text { Female } \\
n=279(55.8 \%)\end{array}$ & $P$-value \\
\hline $\begin{array}{l}\text { Fasting Blood Glucose } \\
\qquad \begin{array}{l}<100 \\
100-125 \\
>125\end{array}\end{array}$ & $\begin{array}{l}(\mathrm{n}=373) \\
75(20.11 \%) \\
99(26.54 \%) \\
199(53.35 \%)\end{array}$ & $\begin{array}{l}(n=151) \\
28(18.54 \%) \\
36(23.84 \%) \\
87(57.62 \%)\end{array}$ & $\begin{array}{l}(n=222) \\
47(21.17 \%) \\
63(28.38 \%) \\
112(50.45 \%)\end{array}$ & 0.3924 \\
\hline $\begin{array}{l}\text { Non-Fasting Blood Glucose } \\
\quad<200 \\
\quad>200\end{array}$ & $\begin{array}{l}(n=459) \\
327(71.24 \%) \\
132(28.76 \%)\end{array}$ & $\begin{array}{l}(n=200) \\
136(68.00 \%) \\
64(32.00 \%)\end{array}$ & $\begin{array}{l}(n=259) \\
191(73.75 \%) \\
68(26.25 \%)\end{array}$ & 0.1775 \\
\hline $\begin{array}{r}\mathrm{HbA} 1 \mathrm{c} \\
\quad<7 \% \\
\geq 7 \%\end{array}$ & $\begin{array}{l}(n=354) \\
139(39.27 \%) \\
215(60.73 \%)\end{array}$ & $\begin{array}{l}(n=150) \\
46(30.67 \%) \\
104(69.33 \%)\end{array}$ & $\begin{array}{l}(n=204) \\
93(45.59 \%) \\
111(54.41 \%)\end{array}$ & 0.0045 \\
\hline $\begin{array}{l}\text { Total cholesterol } \\
\text { Optimal } \\
\text { Borderline High } \\
\text { High }\end{array}$ & $\begin{array}{l}(n=358) \\
217(60.61 \%) \\
82(22.91 \%) \\
59(16.48 \%)\end{array}$ & $\begin{array}{l}(n=153) \\
102(66.67 \%) \\
33(21.57 \%) \\
18(11.76 \%)\end{array}$ & $\begin{array}{l}(n=205) \\
115(56.10 \%) \\
49(23.90 \%) \\
41(20.00 \%)\end{array}$ & 0.0663 \\
\hline $\begin{array}{l}\text { HDL } \\
\text { Optimal } \\
\text { Borderline Low } \\
\text { Low }\end{array}$ & $\begin{array}{l}(\mathrm{n}=337) \\
41(12.17 \%) \\
169(50.15 \%) \\
127(37.69 \%)\end{array}$ & $\begin{array}{l}(n=139) \\
17(12.23 \%) \\
55(39.57 \%) \\
67(48.20 \%)\end{array}$ & $\begin{array}{l}(n=198) \\
24(12.12 \%) \\
114(57.58 \%) \\
60(30.30 \%)\end{array}$ & 0.0022 \\
\hline $\begin{array}{l}\text { LDL } \\
\text { Optimal } \\
\text { Near Optimal } \\
\text { Borderline High } \\
\text { High } \\
\text { Very High }\end{array}$ & $\begin{array}{l}(n=341) \\
122(35.78 \%) \\
102(29.91 \%) \\
67(19.65 \%) \\
29(8.50 \%) \\
21(6.16 \%)\end{array}$ & $\begin{array}{l}(n=141) \\
64(45.39 \%) \\
39(27.66 \%) \\
22(15.60 \%) \\
8(5.67 \%) \\
8(5.67 \%)\end{array}$ & $\begin{array}{l}(n=200) \\
58(29.00 \%) \\
63(31.50 \%) \\
45(22.50 \%) \\
21(10.50 \%) \\
13(6.50 \%)\end{array}$ & 0.0268 \\
\hline $\begin{array}{l}\text { Triglycerides } \\
\text { Optimal } \\
\text { Borderline High } \\
\text { High } \\
\text { Very High }\end{array}$ & $\begin{array}{l}(n=353) \\
218(61.76 \%) \\
68(19.26 \%) \\
62(17.56 \%) \\
5(1.42 \%)\end{array}$ & $\begin{array}{l}(n=149) \\
93(62.42 \%) \\
27(18.12 \%) \\
26(17.45 \%) \\
3(2.01 \%)\end{array}$ & $\begin{array}{l}(n=204) \\
125(61.27 \%) \\
41(20.10 \%) \\
36(17.65 \%) \\
2(0.98 \%)\end{array}$ & 0.8390 \\
\hline $\begin{array}{l}\text { Hemoglobin } \\
\text { Low } \\
\text { Normal } \\
\text { High }\end{array}$ & $\begin{array}{l}(\mathrm{n}=308) \\
90(29.22 \%) \\
214(69.48 \%) \\
4(1.30 \%)\end{array}$ & $\begin{array}{l}(n=129) \\
43(33.33 \%) \\
84(65.12 \%) \\
2(1.55 \%)\end{array}$ & $\begin{array}{l}(n=179) \\
47(26.26 \%) \\
130(72.63 \%) \\
2(1.12 \%)\end{array}$ & 0.3676 \\
\hline $\begin{array}{l}\text { White blood cells } \\
\qquad \begin{array}{l}\text { Low } \\
\text { Normal } \\
\text { High }\end{array}\end{array}$ & $\begin{array}{l}(n=254) \\
2(0.79 \%) \\
216(85.04 \%) \\
36(14.17 \%)\end{array}$ & $\begin{array}{l}(n=108) \\
1(0.93 \%) \\
96(88.89 \%) \\
11(10.19 \%)\end{array}$ & $\begin{array}{l}(n=146) \\
1(0.68 \%) \\
120(82.19 \%) \\
25(17.12 \%)\end{array}$ & 0.2892 \\
\hline
\end{tabular}

HbA1c: Hemoglobin A1c; HDL: High-Density Lipoprotein; LDL: Low-Density Lipoprotein.

vs. $46.61 \%, P<0.0001)$. In addition, female patients had statistically significant higher rates of stroke $(P=0.0124)$, hypercholesterolemia $(P=0.0277)$, non-alcoholic fatty liver disease $(P=0.0439)$, thyroid disease/nodules $(P<$ $0.0001)$, and urinary tract infections (UTIs) $(P=0.0004)$.

The lab results of the patient population are presented in Table 4 . Only $20.11 \%$ of the patients who had fasting blood glucose measurements were within the normal range $(<100)$, and the male patients had a slightly higher proportion in the high range $(>125)$ than the female patients (57.62\% vs. $50.45 \%)$. Nearly twothirds $(60.73 \%)$ of patients had uncontrolled diabetes, as demonstrated by $\mathrm{HbA} 1 \mathrm{c}$ measurements greater than or equal to $7 \%$. In addition, male patients had a statistically significant higher proportion of high $\mathrm{HbA1c}$ than female (69.33\% vs. $54.41 \%, P=0.0045)$. Female patients had statistically significant lower rates of low $\operatorname{HDL}(P=0.0022)$; however, male patients had statistically significant lower rates of high LDL $(P=0.0268)$.

The most common symptoms experienced by the patient population are presented in Table 5 . In general, males reported more symptoms; the only symptoms that were more common in women were neurologic symptoms (cramps, headache, dizziness, and limbs 
Table 5: Symptoms of INDEN ambulatory patients, 2018.

\begin{tabular}{|l|l|l|l|l|}
\hline & Total Population & Male & Female & P-value \\
\hline E = 500 & $\mathbf{n = 2 2 1 ( 4 4 . 2 \% )}$ & n= 279 (55.8\%) & 76 (27.24\%) & 0.0243 \\
\hline Excessive urination & $157(31.40 \%)$ & $81(36.65 \%)$ & $75(26.88 \%)$ & 0.0873 \\
\hline Excessive hunger & $150(30.00 \%)$ & $75(33.94 \%)$ & $22(7.89 \%)$ & 0.3278 \\
\hline Cramps & $45(9.00 \%)$ & $23(10.41 \%)$ & $100(35.84 \%)$ & 0.8162 \\
\hline Headache & $177(35.40 \%)$ & $77(34.84 \%)$ & $110(39.34 \%)$ & 0.0447 \\
\hline Dizziness & $178(35.60 \%)$ & $68(30.77 \%)$ & $89(31.90 \%)$ & 0.2488 \\
\hline Painful urination & $149(29.80 \%)$ & $60(27.15 \%)$ & $16(5.73 \%)$ & 0.7790 \\
\hline Nighttime urination & $30(6.00 \%)$ & $14(6.33 \%)$ & $78(27.96 \%)$ & 0.2197 \\
\hline Amputation & $151(30.20 \%)$ & $73(33.03 \%)$ & $2(0.72 \%)$ & 0.0111 \\
\hline Eye diseases & $11(2.20 \%)$ & $9(4.07 \%)$ & $46(16.49 \%)$ & 0.0006 \\
\hline Limbs Asleep & $111(22.20 \%)$ & $65(29.41 \%)$ & $80(28.67 \%)$ & 0.1256 \\
\hline Lack of Energy & $130(26.00 \%)$ & $50(22.62 \%)$ & $45(16.13 \%)$ & 0.3996 \\
\hline Weight Loss & $87(17.40 \%)$ & $42(19.00 \%)$ & $57(20.43 \%)$ & $18(6.45 \%)$ \\
\hline Foot ulcer & $119(23.80 \%)$ & $62(28.05 \%)$ & $25(11.31 \%)$ & 0.0468 \\
\hline
\end{tabular}

asleep). Male patients had statistically significant higher rates of excessive urination $(P=0.0243)$, amputation $(P=0.0111)$, eye disease $(P=0.0006)$, weight loss $(P=$ 0.0468), and foot ulcers $(P=0.0542)$. Female patients had statistically significant higher rates of headache $(P$ $=0.0447$ ).

\section{Discussion}

This retrospective chart review presents important information about the ambulatory diabetic population of the Dominican Republic, including differences by sex. As expected, a large majority of the diabetic patients had Type 2 diabetes. We found significant differences in demographic, behavioral, and clinical characteristics between male and female patients that can be beneficial in both clinical care and preventive interventions. A previous study of hospitalized diabetes patients from the diabetic foot clinic at INDEN found similar results [9].

Other studies of diabetes in Latin American and Caribbean populations have reported differences in the prevalence of diabetes among men and women [9-13]. One study found Caribbean women to be more likely than men to have uncontrolled diabetes based on $\mathrm{HbA1C}$ and fasting blood glucose [12]. We found that men were more likely to have uncontrolled diabetes based on these factors. However, the majority of both men and women had high $\mathrm{HbA1c}$ and fasting blood glucose levels, suggesting the need for improved disease management overall. However, approximately onethird of the patients were newly diagnosed. In addition, almost one-third of the patients received some form of insulin, indicating appropriate medical treatment.

Comorbidities present a challenge to the burden of diabetes, especially financially. Our findings show a higher prevalence of hypertension in women than in men, consistent with other research from the DR $[9,11]$. Furthermore, this is consistent with a previous study showing that Latin American women over 50 have a higher prevalence of hypertension than men, due to the increased risk of cardiovascular disease in post-menopausal women [14]. In addition, an important modifiable risk factor for Type 2 diabetes, especially among Caribbean women, is obesity $[12,15]$. Obesity negatively impacts other comorbidities, like hypertension, cardiovascular disease and stroke [1]. In our patient population, over three-quarters of the female patients were obese or overweight.

The patient population overall showed high rates of common risk factors for Type 2 diabetes, including lack of exercise. Interventions targeting these risk factors in both men and women could have a positive impact in both prevention of Type 2 diabetes and improving the burden of disease in those already diagnosed. In addition, some risk factors differed significantly for men and women, including lack of exercise and alcohol consumption. Our findings show that more men exercised than women which is consistent with previous research [12]. These findings indicate that gender-specific diabetes-related interventions could be beneficial, to address the different behaviors and disease characteristics between men and women. Tailored interventions would help create equity in healthcare services by ensuring that each gender receives the specific education and information to maximize their potential benefit.

Studies have shown that management of Type 2 diabetes is improved with diabetes health education [16]. An increase in educational programs that emphasize seeking medical attention early could help with both reducing the burden of the disease and decreasing the financial burden due to comorbidities. 
The high proportion of uncontrolled diabetes, in combination with the obesity rates and the lack of exercise, suggests the need for effective programs to address not only diabetes prevention, but diabetes management for those already diagnosed. Almost half of the patients visited the clinic 3 or more times per year, providing an opportunity for effective diabetes education within the clinic. In addition, nearly threequarters of the patients reported having a first degree relative with diabetes; this suggests that communitybased interventions that involve the entire family can be beneficial in both disease management and spreading awareness about prevention to children. A communitybased health program that utilized trained community workers to educate an Hispanic population in the United States was found to be successful at increasing the knowledge about Type 2 diabetic risk factors and control and prevention [17]. In addition, a communitybased educational study in rural DR aimed at lifestyle modification was successful in improving $\mathrm{HbA1c}$ and reducing cardiovascular risk [18]. The program trained community leaders about nutrition, weight loss, diabetes, and physical activity. After a 1-year period of group meetings led by the community leaders, there was a statistically significant decrease in the average $\mathrm{HbA1C}$ and blood pressure of the population [18]. In addition, a community-based approach to healthcare may enhance the community's trust in the healthcare system [19].

Finally, the social determinants of health should also be addressed for effective diabetes prevention and control in the DR. Diabetic medications can be a significant financial burden [18]. Many Dominicans who live in rural areas don't have refrigerators to keep insulin cold, resulting in improper disease management. In addition, some neighborhoods are unsafe, limiting the ability for residents to get exercise, particularly women. Patients without sufficient access to healthcare services and medications are at a higher risk for diabetes-related complications [20]. Social and economic determinants of health such as poverty, rural location, and type of neighborhood contribute to the inequitable distribution of the disease in the Dominican population. Numerous governmental [21] and non-governmental [22] programs in the Dominican Republic target poverty and its effects; these need to be expanded to reach a greater proportion of the population. Future research on how social and economic factors impact all types of diabetes in the Dominican population would be beneficial.

One limitation of this study was its retrospective design. Some charts were missing data for one or more variables. Records for patients who had had only initial visits were lacking lab results, because they had not returned for their next appointment. In addition, some data may have been inaccurately recorded due to errors in translation. Finally, social acceptability bias may have resulted in underreporting of smoking and/or alcohol use by some patients.

In conclusion, our findings indicate that there are important clinical and behavioral differences by sex among patients with diabetes (predominantly Type 2) in the DR, as well as high rates of important risk factors. Gender-specific interventions for patients with diabetes about the importance of early medical care and the impact of lifestyle on risk factors can help to minimize the burden of the disease and improve the quality of life for the diabetic population. In addition, community-based programs focusing on the prevention of high prevalence risk factors, like obesity and hypertension, may help to slow the spread of Type 2 diabetes, improve the health of the population, and decrease the economic burden of disease on both the population and the healthcare system of the Dominican Republic.

\section{Acknowledgments}

We would like to thank Dr. Marcos Nuñez, Dean of the School of Medicine of UNIBE, for establishing this research collaboration; Jose Lugo Vargas and Selena Moore who helped in data abstraction; and the University of Virginia's Center for Global Health University Scholars program for funding the project.

\section{Authors Contribution}

All co-authors have contributed to and agree with the contents of the manuscript.

\section{References}

1. International Diabetes Federation (2019) IDF Diabetes Atlas. ( $9^{\text {th }}$ edn $)$.

2. Aschner P, Aguilar-Salinas C, Aguirre L, Franco L, Gagliardino JJ, et al. (2014) Diabetes in south and central America: An update. Diabetes Res Clin Pract 103: 238-243.

3. Ibrahim A, Camilo A, Cepeda J, Ruiz Matuk C (2018) Caracterización de la Diabetes Mellitus en la República Dominicana. UNIBE.

4. World Health Organization (2016) WHO Diabetes country profiles: Dominican Republic.

5. Sepulveda J, Murray C (2014) The state of global health in 2014. Science 345: 1275-1278

6. Barcelo A, Aedo C, Rajpathak S, Robles S (2003) The cost of diabetes in Latin America and the Caribbean. Bull World Health Organ 81: 19-27.

7. Aponte J, Boutin-Foster C, Alcantara R (2012) Knowledge, perceptions, and experiences of Dominicans with diabetes. J Immigr Minor Health 14: 1006-1013.

8. Canario JA, Lizardo J, Espinal R, Colome M (2016) Gaps in health research in the Dominican Republic. Rev Panam Salud Publica 39: 179-185.

9. Bonilla GA, Hornsby PP, Pannone AF, Case SK, Aviles ES, et al. (2019) Demographic and clinical characteristics of dominican adults admitted to a diabetic foot clinic in the Dominican Republic, 2015. Diabetes Metab Syndr 13: $1727-1732$

10. Irazola V, Rubinstein A, Bazzano L, Calndrelli M, ChungShiuan C, et al. (2017) Prevalence, awareness, treatment 
and control of diabetes and impaired fasting glucose in the southern cone of Latin America. PLoS One 12: e0183953.

11. Acosta D, Rottbeck R, Rodriguez JG, Gonzalez LM, Almanzar MR, et al. (2010) The prevalence and social patterning of chronic diseases among older people in a population undergoing health transition. A 10/66 Group cross-sectional population-based survey in the Dominican Republic. BMC Public Health 10: 344.

12. Sobers-Grannum N, Murphy MM, Nielson A, Guell C, Samuels TA, et al. (2015) Female gender is a social determinant of diabetes in the Caribbean: A systematic review and meta-analysis. PLoS One 10: e0126799.

13. Bennett NR, Francis DK, Ferguson TS, Hennis AJM, Wilks RJ, et al. (2015) Disparities in diabetes mellitus among Caribbean populations: A scoping review. Int J Equity Health 14: 23.

14. Rubinstein AL, Irazola VE, Calandrelli M, Chung-Shiuan C, Gutierrez L, et al. (2016) Prevalence, awareness, treatment, and control of hypertension in the southern cone of Latin America. Am J Hypertens 29: 1343-1352.

15. Tucker KL, Bermudez OI, Castaneda C (2000) Type 2 diabetes is prevalent and poorly controlled among Hispanic elders of Caribbean origin. Am J Public Health 90: 12881293.
16. Ezenwaka C, Offiah N (2002) Patient's health education and diabetes control in a developing country. Acta Diabetol 40: $173-175$

17. Cruz Y, Hernandez-Lane M, Cohello JI, Bautista CT (2013) The effectiveness of a community health program in improving diabetes knowledge in the Hispanic population: Salud y bienestar (Health and Wellness). J Community Health 38: 1124-1131.

18. West-Pollak A, Then EP, Podesta C, Hedelt A, Perry ML, et al. (2014) Impact of a novel community-based lifestyle intervention program on type 2 diabetes and cardiovascular risk in a resource-poor setting in the Dominican Republic. Int Health 6: 118-124.

19. Sastre F, Rojas P, Cyrus E, De La Rosa M, Khoury AH (2014) Improving the health status of Caribbean people: Recommendations from the Triangulating on Health Equity summit. Glob Health Promot 21: 19-28.

20. Mach JC, Barone H, Boni C, Jimenez H, Tinglin M (2019) Evaluating the impact of an international short-term medical mission through diabetic glycemic control. J Public Health (Oxf) 41: 815-820.

21. The World Bank (2017) Including the Excluded: Improving Social Protection Services in the Dominican Republic.

22. (2020) Partners for Rural Health in the Dominican Republic. 